\title{
Phylogeny of the Mycoplasma mycoides cluster based on analysis of five conserved protein-coding sequences and possible implications for the taxonomy of the group
}

\author{
Lucía Manso-Silván, ${ }^{1}$ Xavier Perrier ${ }^{2}$ and François Thiaucourt ${ }^{1}$ \\ ${ }^{1}$ CIRAD BIOS, UPR15, Control of Exotic and Emerging Animal Diseases, TA A-15/G, Campus \\ International Baillarguet, 34398 Montpellier Cedex 5, France \\ ${ }^{2}$ CIRAD BIOS, UPR75, Biomathematics, Genetic Improvement of Vegetatively Propagated Crops, \\ TA 71/09, Campus Lavalette, 34398 Montpellier Cedex 5, France
}

Correspondence

Lucía Manso-Silván

lucia.manso-silvan@cirad.fr

\begin{abstract}
A phylogenetic tree of the Mycoplasma mycoides cluster was inferred from a set of concatenated sequences from five housekeeping genes ( $f u s A, g l p Q, g y r B$, lepA and $r p o B$ ). The relevance of this phylogeny was reinforced by detailed analysis of the congruence of the phylogenies derived from each of the five individual gene sequences. Two subclusters were distinguished. The M. mycoides subcluster comprised M. mycoides subsp. mycoides biotypes Small Colony (SC) and Large Colony (LC) and M. mycoides subsp. capri. The latter two groups could not be clearly separated, which supports previous proposals that they be united into a single taxonomic entity. The Mycoplasma capricolum subcluster included M. capricolum subsp. capricolum, M. capricolum subsp. capripneumoniae and Mycoplasma sp. bovine group 7 of Leach, a group of strains that remains unassigned. This group constituted a distinct branch within this cluster, supporting its classification as a subspecies of M. capricolum. Mycoplasma cottewii and Mycoplasma yeatsii clustered in a group that was distinct from Mycoplasma putrefaciens and they were all clearly separated from the M. mycoides cluster. In conclusion, this approach has allowed us to assign phylogenetic positions to all members of the M. mycoides cluster and related species and has proved the need to adjust the existing taxonomy. Furthermore, this method may be used as a reference technique to assign an unequivocal position to any particular strain related to this cluster and may lead to the development of new techniques for rapid species identification.
\end{abstract}

\section{INTRODUCTION}

The Mycoplasma mycoides cluster, as described by Cottew et al. (1987), represents a group of six closely related mycoplasmas that are pathogenic for ruminants and which are currently referred to as Mycoplasma mycoides subsp. mycoides Small Colony biotype (MmmSC) and Large Colony biotype (MmmLC), M. mycoides subsp. capri

\footnotetext{
Abbreviations: CBPP, contagious bovine pleuropneumonia; CCPP, contagious caprine pleuropneumonia; MBG7, Mycoplasma sp. bovine group 7 of Leach; Mcc, M. capricolum subsp. capricolum; Mccp, M. capricolum subsp. capripneumoniae; Mmc, M. mycoides subsp. capri; MmmLC, M. mycoides subsp. mycoides Large Colony biotype; MmmSC, M. mycoides subsp. mycoides Small Colony biotype.

The GenBank/EMBL/DDBJ accession numbers of the partial fus $A$, $g / p Q, g y r B$, lep $A$ and $r p o B$ gene sequences of 26 strains analysed here are listed in Supplementary Table S1.
}

Accession numbers of the sequences used in this study are available with the online version of this paper.
(Mmc), Mycoplasma capricolum subsp. capricolum (Mcc), M. capricolum subsp. capripneumoniae (Mccp) and Mycoplasma sp. bovine group 7 of Leach (MBG7). In addition, on the basis of $16 \mathrm{~S}$ rRNA gene sequence similarity, other species such as the pathogen Mycoplasma putrefaciens (Weisburg et al., 1989) and the related saprophytic species Mycoplasma cottewii and Mycoplasma yeatsii (Heldtander et al., 1998) have been included in the phylogenetic $M$. mycoides cluster, within the Spiroplasma group.

MmmSC and Mccp are the causative agents of contagious bovine and contagious caprine pleuropneumonia (CBPP and CCPP), respectively, which are diseases of major concern, classed by the World Organization for Animal Health (OIE) as notifiable animal diseases. Other members of the cluster (MmmLC, Mmc and $\mathrm{Mcc}$ ) and $M$. putrefaciens are involved in the 'MAKePS' syndrome, characterized by mastitis, arthritis, keratoconjunctivitis, pneumonia and septicaemia in small ruminants (Thiaucourt \& Bölske, 1996). Finally, MBG7 represents a 
group of strains that are serologically distinct from other bovine mycoplasmas (Leach, 1967) and which cause mastitis and polyarthritis in cattle.

Because the different members of the M. mycoides cluster share many genotypic and phenotypic traits, their classification and evolutionary relationships have been difficult to establish. This may explain why the group of strains known as MBG7 remains unassigned, while Mccp was named only relatively recently (Leach et al., 1993). Initial classification within this cluster was attempted based on DNA-DNA hybridization and biochemical and serological methods as well as 2D PAGE patterns (Cottew et al., 1987), which resulted in differentiation of the M. mycoides subspecies on one side and the $M$. capricolum subspecies on the other. The position of MBG7 was controversial due to conflicting data obtained from the various methods applied and remains unresolved, though recent studies concur in favour of classification of this group of strains within a subspecies of $M$. capricolum (Harasawa et al., 2000; Pettersson et al., 1996; Thiaucourt et al., 2000).

Another controversial issue is the distinction of MmmLC and $\mathrm{Mmc}$ as separate subspecies: numerous investigations, based on both genetic and antigenic analyses, suggest that they may be classed together as a single subspecies within the M. mycoides subcluster (Costas et al., 1987; Leach et al., 1989; Monnerat et al., 1999; Olsson et al., 1990; Rodwell, 1982; Salih et al., 1983; Taylor et al., 1992; Vilei et al., 2006).

The phylogeny of the M. mycoides cluster inferred from $16 \mathrm{~S}$ rRNA gene sequences (Pettersson et al., 1996) relied on too few nucleotide variations to construct a reliable phylogenetic tree. It was then proposed that further studies be based on sequence analysis of alternative genes. To this aim, five housekeeping genes have been identified ( $f u s A, g l p Q$, gyrB, lepA and $r p o B$ ), taking into consideration the requirements for phylogenetic studies described by Woese (1987). Phylogenetic analysis based on concatenated sequences from these five genes and reinforced by separate analysis of each of the five genes has enabled us to determine evolutionary relationships between all the members of the M. mycoides cluster.

\section{METHODS}

Determination of partial gene sequences. A sample of strains from various geographical origins was chosen carefully, including representatives of all members of the $M$. mycoides cluster and related species and adapting the number of strains to the known variability within each group. The 28 strains analysed are listed in Table 1, with reference to their origin. The strains were characterized according to results obtained using two different techniques: growth inhibition with hyperimmune sera (Clyde, 1983) and dot immunobinding (Poumarat et al., 1992).

Mycoplasma strains were cultured in modified Hayflick's broth [PPLO broth without crystal violet $\left(21 \mathrm{~g} \mathrm{l}^{-1}\right), 20 \%$ horse serum decomplemented for $1 \mathrm{~h}$ at $56{ }^{\circ} \mathrm{C}, 5 \%$ fresh yeast extract, $0.2 \%$ glucose, $0.4 \%$ sodium pyruvate] at $37{ }^{\circ} \mathrm{C}$ in $5 \% \mathrm{CO}_{2}$ and were harvested in the late exponential phase of growth. Preparation of PCR templates in lysis buffer was based on the method of Miserez et al. (1997).

The selection of primer pairs for amplification of fusA, $g l p Q, g y r B$, lep $A$ and $r p o B$ sequences (Table 2) was based on initial alignments of sequences obtained from published mycoplasma genomes (http:// cbi.labri.fr/outils/molligen/home.php) as well as from ongoing genome sequencing projects (MmmLC strain 95010-C1 and MmmSC strain 8740-Rita). Amplification of each of the target sequences was performed in $50 \mu \mathrm{l}$ reactions containing $1 \times$ Taq buffer (Qiagen) (giving a final $\mathrm{MgCl}_{2}$ concentration of $1.5 \mathrm{mM}$ ), $150 \mu \mathrm{M}$ dCTP and dGTP, $300 \mu \mathrm{M}$ dATP and TTP (dNTPs from Roche), $0.4 \mu \mathrm{M}$ each of the corresponding forward and reverse primer, $1 \mathrm{U}$ Taq polymerase (Qiagen) and $1 \mu \mathrm{l}$ sample. The PCR thermal program consisted of an initial denaturation step of $2 \mathrm{~min}$ at $94{ }^{\circ} \mathrm{C}$ followed by 35 cycles of $30 \mathrm{~s}$ at $94{ }^{\circ} \mathrm{C}, 30 \mathrm{~s}$ at $52{ }^{\circ} \mathrm{C}$ and $45 \mathrm{~s}$ at $72{ }^{\circ} \mathrm{C}$ and a final extension step of $5 \mathrm{~min}$ at $72{ }^{\circ} \mathrm{C}$. An exception was made for the PCR with primers GlpQ-L-F/-R, for which the annealing temperature was $45{ }^{\circ} \mathrm{C}$, with the reaction conditions otherwise remaining the same.

The primer pairs listed in Table 2 were also used for sequencing of the corresponding PCR products by Genome Express (Meylan, France). Sequences were aligned using the AlignX tool of Vector NTI 9.0 (Invitrogen) and they were trimmed to the same size, providing fragments of $561 \mathrm{bp}(f u s A), 538 \mathrm{bp}(g l p Q), 533 \mathrm{bp}$ (gyrB), $657 \mathrm{bp}$ (lepA) and $625 \mathrm{bp}($ rpoB) for phylogenetic analyses. No deletions/ insertions were observed except for the $g l p Q$ sequences of the two $M$. putrefaciens strains, which contained an extra codon. The partial nucleotide sequences determined in this study for each of the five protein-coding genes from 24 strains, as well as from MmmSC strain 8740-Rita and MmmLC strain 95010-C1, were deposited in GenBank under the accession numbers shown in Supplementary Table S1 (available in IJSEM Online). Sequences of these five genes were also retrieved from the published genomes of the type strains MmmSC $\mathrm{PG}^{\mathrm{T}}$ (GenBank accession no. BX293980) and Mcc California kid ${ }^{\mathrm{T}}$ (CP000123). A total of 28 sequences were therefore available for each of the five genes studied.

Phylogenetic analyses. Sequence analyses were conducted using MEGA 3.1 (Kumar et al., 2004; http://www.megasoftware.net), whereas Darwin 5.0 (Perrier et al., 2003; http://darwin.cirad.fr/darwin) was used for diversity analyses. Phylogenetic trees were constructed using the neighbour-joining algorithm (Saitou \& Nei, 1987) based on pairwise dissimilarities between strains. Since other methods such as minimum evolution and maximum parsimony by close-neighbour interchange (MEGA 3.1) generated nearly identical topologies, the neighbour-joining method was retained for its properties of convergence and consistency, as well as low computational complexity.

Pairwise distances were calculated as the proportion of non-matching sites between pairs of sequences. The three positions corresponding to an extra codon in the $g l p Q$ sequences of the M. putrefaciens strains were deleted to avoid gaps. Since the sequences were highly similar, the effect of multiple substitutions was considered negligible and no correction was applied to dissimilarities. The mean net distance between groups was also calculated as the arithmetic mean of the pairwise distances between two groups corrected by the corresponding mean distance within groups (MEGA 3.1).

A bootstrap analysis with 500 replicates was performed to test the stability on randomly chosen sets of positions. To analyse the stability through the sample of strains, the influence of each sequence on the tree structure was estimated using a tool available in Darwin 5.0. In a jackknife-like procedure, each unit is successively removed and a partial tree is inferred from all units except the removed one. This tree is compared to the initial tree using a topological criterion, the quartet tree distance $(\mathrm{Qd})$, which measures among all quartets 
Table 1. Mycoplasma strains analysed in this study

Suppliers of strains are abbreviated as follows: Arist. U., Professor K. Sarris, Aristoteles University, Thessaloniki, Greece; Bern U., Professor J. Nicolet, Institute for Veterinary Bacteriology, University of Bern, Switzerland; BgVV, Dr K. Sachse, Federal Institute for Health Protection of Consumers and Veterinary Medicine, Division 4, Jena, Germany; Cal. U., Dr A. J. DaMassa, Department of Population Health and Reproduction, School of Veterinary Medicine, University of California, Davis, USA; CIRAD, Centre de Coopération Internationale en Recherche Agronomique pour le Développement, UPR-15, Montpellier, France; CIRG, Dr Rajneesh Rana, Central Institute for Research on Goats, Makhdoom, Mathura, India; FDVR, Dr E. P. Lindley, Federal Department of Veterinary Research, Vom, Nigeria; IVRI, Dr V. P. Singh, Indian Veterinary Research Institute, Izatnagar, India; LANAVET, Dr A. Martrenchar, Laboratoire National Vétérinaire, Garoua, Cameroon; LPA, Dr J. Domenech, Laboratoire Central de Pathologie Animale, Bingerville, Ivory Coast; NVI-E, Dr Y. Fikré, National Veterinary Institute, Debre Zeit, Ethiopia; NVI-S, Dr G. Bölske, National Veterinary Institute, Uppsala, Sweden; TiHo, Dr R. Schmidt, Tierärztliche Hochschule, Hannover, Germany. NK, Not known.

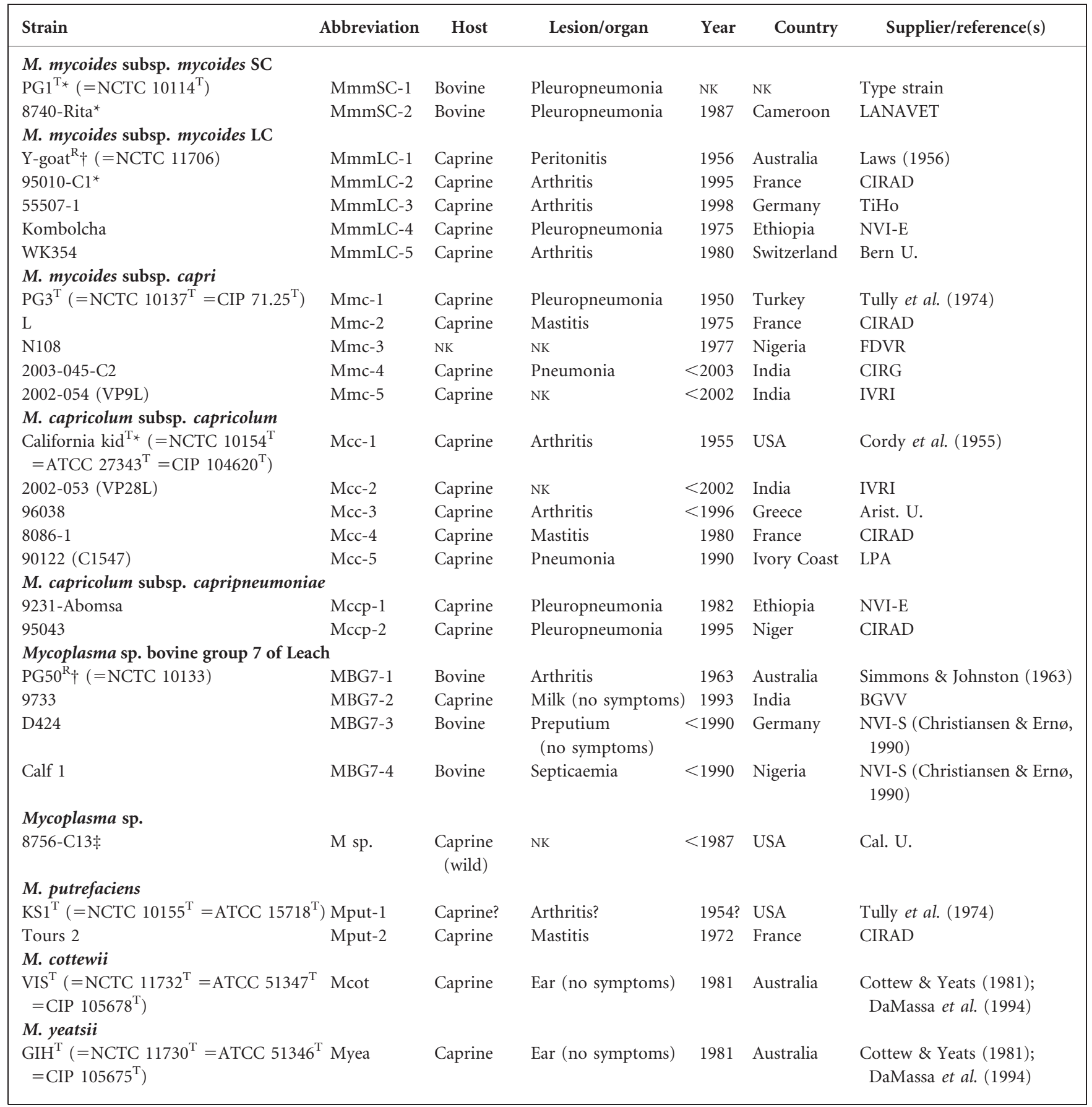

*Strains for which sequences were obtained from published genomes or from ongoing genome-sequencing projects.

$\dagger$ Proposed reference strain for this taxon.

$\ddagger$ The taxonomic position of strain $8756-\mathrm{C} 13$ is uncertain. 
(subsets of four sequences) the proportion of those that do not share the same topology. This distance is regarded as a measure of the influence that the removed sequence has on the tree structure. A high Qd value for a given unit means that this sequence conveys a strong and atypical signal, largely incompatible with the common signal of the other units, and is highly influential on the tree structure. On the other hand, a low Qd value means that the removed unit conveys a signal similar to that of other units and is not particularly influential on the structure. Similar and low Qd values are expected for a coherent set of units. The detection of particularly high Qd values warns of the presence of atypical units. Since tree construction methods are known to be very sensitive to atypical units, sequences with unusual strong influence were excluded in order to infer more reliable trees.

\section{RESULTS AND DISCUSSION}

\section{Phylogenetic approach}

Five conserved protein-coding genes, presenting greater variability than $16 \mathrm{~S}$ rRNA gene sequences, were selected for phylogenetic analysis of the M. mycoides cluster. These genes were located at distant positions, distributed along the genome of sequenced mycoplasmas belonging to this cluster. Four of them are informational genes: fus $A$ encodes the elongation factor G, gyrB encodes the DNA gyrase subunit B, lepA encodes the GTP-binding membrane protein or elongation factor LepA and $r p o B$ encodes the DNAdependent RNA polymerase $\beta$-subunit. These universally conserved genes have been selected as complementary to $16 \mathrm{~S}$ rRNA gene analysis for bacterial identification and phylogenetic studies (Santos \& Ochman, 2004) and $r p o B$ has already been used for phylogenetic analysis of Mycoplasma species (Kim et al., 2003; Vilei et al., 2006). An operational gene, $g l p Q$, was also included. It encodes glycerophosphoryl diester phosphodiesterase and has also been used to define prokaryote taxa (Schwan et al., 2005).

Assuming that the evolutionary history of organismal lineages in the M. mycoides cluster can be properly estimated in the form of a phylogenetic tree, it was proposed that this 'species tree' may be inferred from the phylogenies based on the five selected genes, the 'gene trees'. However, it is now well established that different genes sampled from the same set of taxa can produce markedly different phylogenies because of limited variability, inadequate sampling or random convergence, but also because of biological phenomena related to evolutionary events such as horizontal transfer or selection pressure. The problem is then to reconcile the trees derived from different genes to approximate the species tree. Two approaches are generally proposed: consensus methods and total evidence. The former (strict consensus, relaxed consensus or, more recently, consensus networks) considers discordance as uncertainty and produces trees that are often largely unresolved. In contrast, the total evidence approach infers a tree from a set of concatenated gene sequences assuming that each gene tree contains, at least in part, a common phylogenetic signal that will emerge with sufficient sampling. It has been shown that total evidence is often efficient in overcoming the incongruences present in single-gene analyses (Rokas et al., 2003). Therefore, the approach chosen in this study was to infer the phylogeny of the M. mycoides cluster from a sequence generated by concatenation headto-tail of the five selected gene sequences. However, since the expected statistical smoothing is not guaranteed with only five genes, the confidence attached to the inferred phylogeny would be strengthened if the common structure could be retrieved, at least at the level of the main subclusters, in each of the individual gene phylogenies or if the biological causes for strong incongruence on a particular gene could be recognized and explained. For this reason, each of the five gene trees was analysed separately and compared to the total evidence tree.

\section{The M. mycoides cluster tree as inferred from a set of five concatenated protein-coding sequences}

Primer pairs for amplification of $f u s A, g l p Q, g y r B$, lepA and $r p o B$ sequences were selected on the basis of homologous regions detected by alignment of the available sequences of the type strains $\mathrm{MmmSC} \mathrm{PGl}^{\mathrm{T}}$ and Mcc California $\mathrm{kid}^{\mathrm{T}}$ and sequences obtained through ongoing genome

Table 2. PCR primers

PCR product sizes and $5^{\prime}$ positions of forward primers for each target were taken from the genome sequences of MmmSC PG1 ${ }^{\mathrm{T}}(\mathrm{GenBank}$ accession no. BX293980) and Mcc California kid ${ }^{\mathrm{T}}$ (CP000123).

\begin{tabular}{|c|c|c|c|c|c|c|}
\hline \multirow[t]{2}{*}{ Target } & \multicolumn{2}{|r|}{ Forward primer } & \multicolumn{2}{|r|}{ Reverse primer } & \multirow{2}{*}{$\begin{array}{c}\text { Product } \\
\text { (bp) }\end{array}$} & \multirow[t]{2}{*}{ Position } \\
\hline & Name & Sequence $\left(5^{\prime}-3^{\prime}\right)$ & Name & Sequence $\left(5^{\prime}-3^{\prime}\right)$ & & \\
\hline fusA & FusA-F & TGAAATTTTTAGATGGTGGAGAA & FusA-R & GGTAATTTAATAGTTTCACGATATGAA & 781 & 680 \\
\hline$g l p Q$ & GlpQ-L-F & GTGAATATAATGATCTTAGTAGC & GlpQ-L-R & TCAGGATAATCTGAAATATAACC & 695 & 1 \\
\hline $\operatorname{gyr} B$ & GyrB-F & GTGTTGTTAATGCTTTATCTTTATATG & GyrB-R & TTGTGGATCTGTATGTCTAACTGATAAAA & 635 & 359 \\
\hline lepA & LepA-L-F & AGAAATTTTAGTATTATTGCTCATA & LepA-L-R & CTTGGAGCAGTTGCAATTAGTTCAA & 1097 & 19 \\
\hline rpoB & RpoB-F2 & GCTCAATCTAATGTTAATCAAGATG & RpoB-R2 & TCTTCTGGAGAAAGTTGAACTTGTC & 824 & 1963 \\
\hline
\end{tabular}


Table 3. Comparison of the variability of different gene sequences analysed among members of the $M$. mycoides cluster and related species

The numbers of variable and phylogenetically informative sites (variables excluding singletons) were calculated from sequence alignments of 28 strains of the M. mycoides cluster and related species listed in Table 1 and from the alignment of the 23 strains belonging to the M. mycoides cluster. M. putrefaciens, M. cottewii and $M$. yeatsii strains were excluded from these latter comparisons, as well as the uncharacterized strain $8756-\mathrm{C} 13$. For strain details see Table 1.

\begin{tabular}{|c|c|c|c|c|c|}
\hline \multirow[t]{2}{*}{ Gene } & \multirow[t]{2}{*}{$\begin{array}{l}\text { Sequence } \\
\text { (bp) }\end{array}$} & \multicolumn{2}{|c|}{$\begin{array}{l}\text { M. mycoides cluster } \\
\text { and related species }\end{array}$} & \multicolumn{2}{|c|}{$\begin{array}{l}\text { M. mycoides } \\
\text { cluster only }\end{array}$} \\
\hline & & $\begin{array}{l}\text { Variable } \\
\text { sites }\end{array}$ & $\begin{array}{l}\text { Informative } \\
\text { sites }\end{array}$ & $\begin{array}{l}\text { Variable } \\
\text { sites }\end{array}$ & $\begin{array}{l}\text { Informa- } \\
\text { tive sites }\end{array}$ \\
\hline fusA & 561 & 106 & 90 & 36 & 29 \\
\hline$g l p Q$ & 538 & 185 & 165 & 55 & 47 \\
\hline gyrB & 533 & 172 & 150 & 52 & 41 \\
\hline lepA & 657 & 182 & 158 & 68 & 51 \\
\hline rpoB & 625 & 149 & 126 & 50 & 35 \\
\hline $\begin{array}{l}\text { Concate- } \\
\text { nated set }\end{array}$ & 2914 & 794 & 686 & 261 & 203 \\
\hline
\end{tabular}

sequencing projects (MmmLC strain 95010-C1 and MmmSC strain 8740-Rita). The primers listed in Table 2 yielded the expected PCR products from all of the 24 additional strains belonging to the $M$. mycoides cluster and related species analysed in this study (Table 1), whereas unrelated mycoplasmas such as Mycoplasma auris $\mathrm{UIA}^{\mathrm{T}}$ failed to yield a PCR product. It must be noted that, as seen by analysis of available, unrelated mycoplasma gene sequences, it is unlikely that these primer pairs will be adequate for PCR amplification from other taxa. In all, 28 sequences were obtained for each of the five genes, which were aligned and trimmed to the same size, providing gene fragments ranging from 533 to $657 \mathrm{bp}$ (Table 3). The five gene fragments corresponding to each of the 28 strains were concatenated head-to-tail, providing a set of sequences of 2914 bp for phylogenetic analysis.

As shown by pairwise distance analysis (Fig. 1), the strains of the M. mycoides cluster were closely related, with maximal divergence limited to $4.6 \%$, whereas strains of $M$ putrefaciens, $M$. cottewii and $M$. yeatsii were easily differentiated from them (14.6-16.3 \% distance). The mean intragroup distance was $0 \%$ for MBG7 and also very limited for MmmSC and Mccp ( $0.1 \%$ dissimilarity), whereas MmmLC and Mmc respectively showed 1.5 and $0.8 \%$ intraspecific divergence. Analysis of the mean net distance between groups revealed a very close identity of MmmLC and Mmc strains ( $0.3 \%$ net distance) and placed MBG7 closer to Mcc (1.9\% net divergence, compared with $3.9 \%$ net distance from MmmSC).

Phylogenetic analysis using the neighbour-joining method resulted in the tree topology shown in Fig. 2. The mycoplasmas from the M. mycoides cluster were arranged in two subclusters, one grouping the $M$. mycoides subspecies and the other comprising the $M$. capricolum subspecies and MBG7 strains. MmmSC strains constituted a separate branch within the $M$. mycoides subcluster, whereas MmmLC and Mmc strains could not be separated clearly from each other. The $M$. capricolum subcluster also comprised two branches, one containing Mcc and Mccp strains and the other represented by MBG7 strains. The uncharacterized strain 8756-C13 (labelled 'M sp.') was

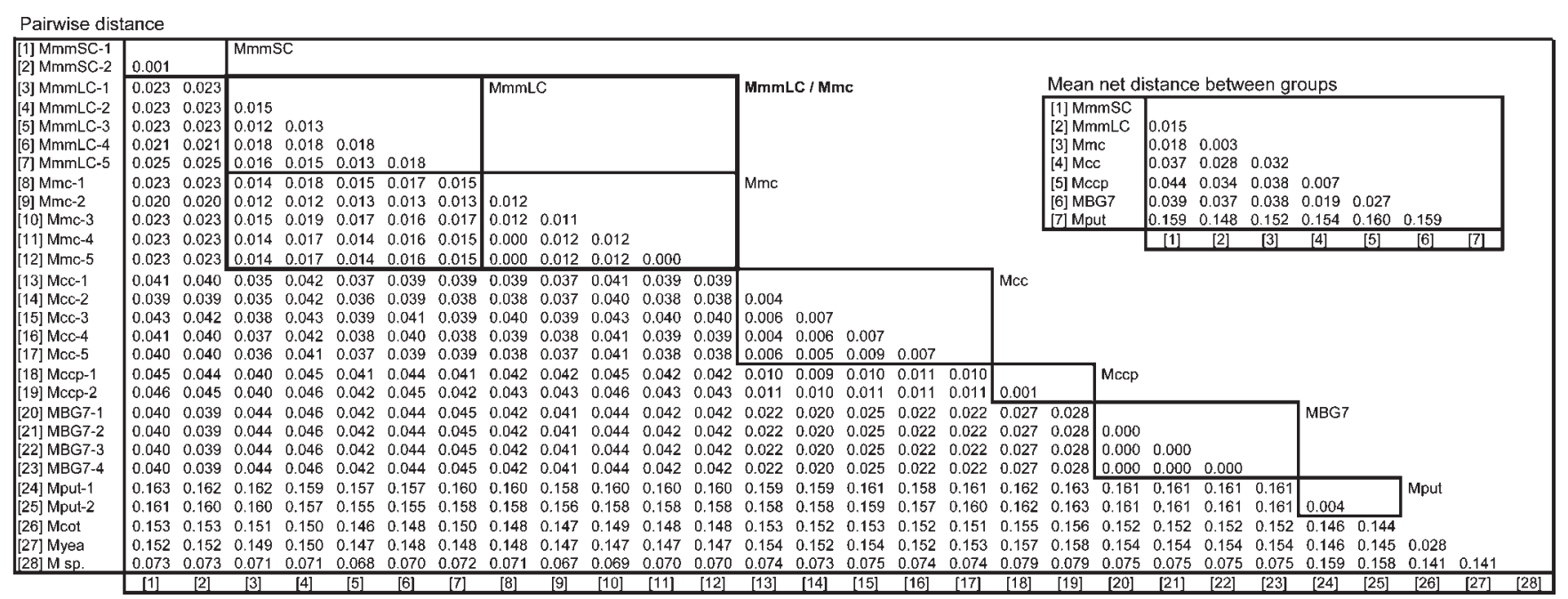

Fig. 1. Pairwise distance analysis of concatenated protein-coding sequences from 28 strains calculated as the proportion of non-matching sites between pairs of sequences. The mean net distances between groups are displayed at the top right. Boxes group the different subspecies, with a bold box grouping M. mycoides subsp. mycoides LC and M. mycoides subsp. capri. For details on strains including abbreviations refer to Table 1. 


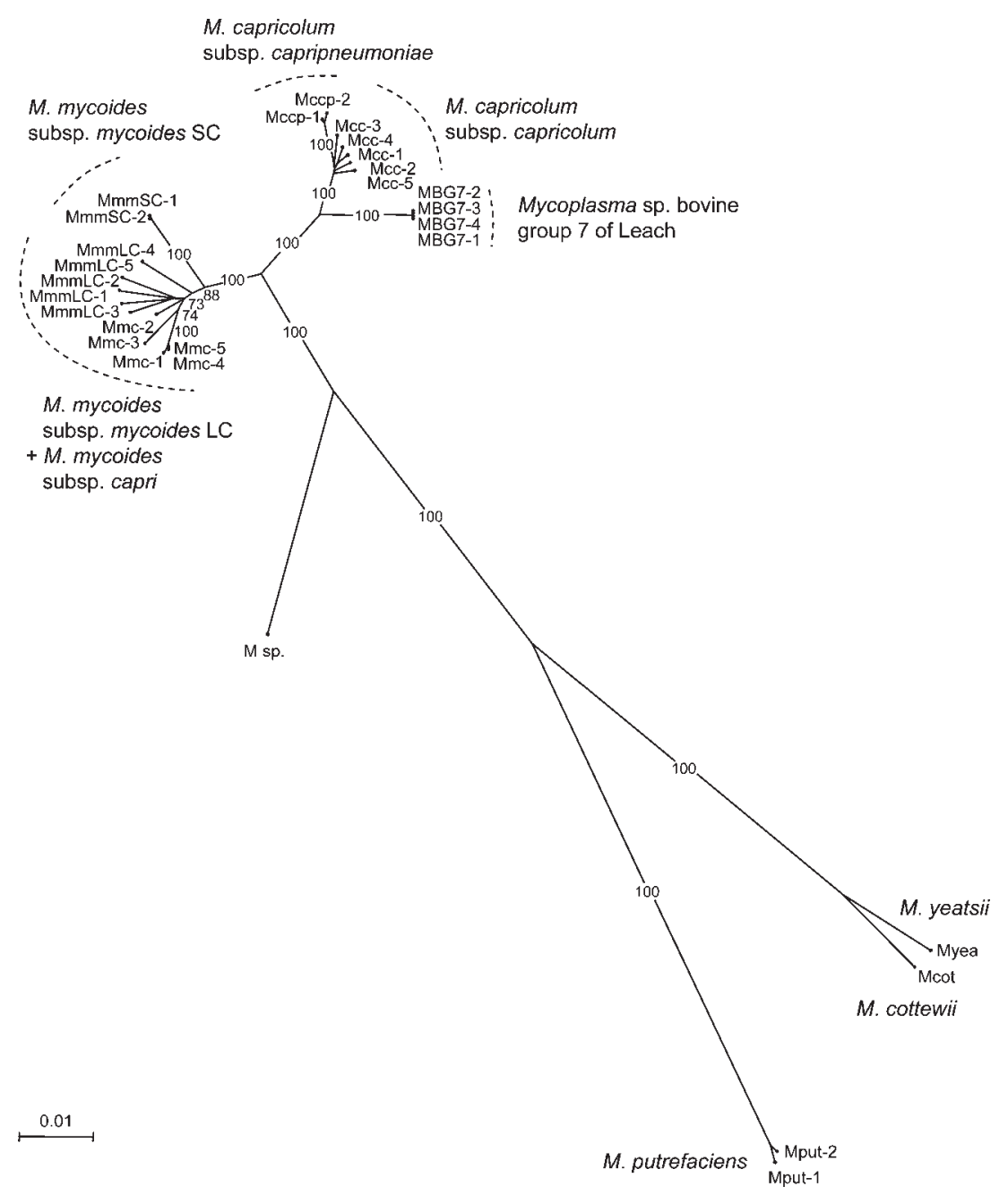

Fig. 2. Phylogenetic tree derived from distance analysis of five concatenated proteincoding sequences (fus $A, g / p Q, \operatorname{gyr} B$, lepA, $r p o B)$. The tree was constructed using the neighbour-joining algorithm. Bootstrap percentage values were calculated from 500 resamplings and values over $70 \%$ are displayed. Bar, distance equivalent to 1 substitution per 100 nucleotide positions. The location of subspecies and groups of strains is indicated. See Table 1 for strain details and Supplementary Table S1 for sequence accession numbers. placed in an intermediate position between the cluster and the other related species. $M$. cottewii and $M$. yeatsii clustered together, separate from $M$. putrefaciens, and they were all clearly separated from the M. mycoides cluster. All the main branches indicated here were supported by maximum bootstrap values $(100 \%)$.

\section{Assessment of the concordance of individual gene trees with the tree based on concatenated sequences}

To assess the adequacy of the phylogeny inferred from concatenated sequences, separate analyses of each of the five genes were performed on 23 strains belonging to the M. mycoides cluster. To facilitate comparisons, the trees were rooted using the uncharacterized strain 8756-C13. However, since atypical sequences such as the outgroup can deeply disturb tree structures, this was not included in the set of analysed strains but was grafted onto the inferred trees afterwards. For the same reason, influential units were investigated systematically by calculating Qd values, as described in Methods. In order to verify the stability of the phylogenetic signal along the gene sequences, separate analyses were performed on sequential subsequences. Finally, independent analyses were also performed on each of the three codon positions, which do not undergo the same evolutionary processes. The first two codon positions are strongly constrained, minimizing the risk of homoplasy, while the third codon position is often free. Modifications in the first two positions tend to result in a change in the amino acid residue. Substitutions in the second codon position were infrequent and were often associated with amino acid changes, accompanied by a change in the first position. The first codon position was therefore retained to avoid this possible redundancy.

The structure of each of the trees based on individual gene sequences was compared to the topology of the tree based on concatenated sequences, focusing comparisons on the distribution of the different subspecies according to the main branches supported by high bootstrap values.

Tree based on fus $A$ sequences. The phylogenetic tree constructed from fusA sequences (Fig. 3a) was in general agreement with the tree based on concatenated sequences. 
(a)

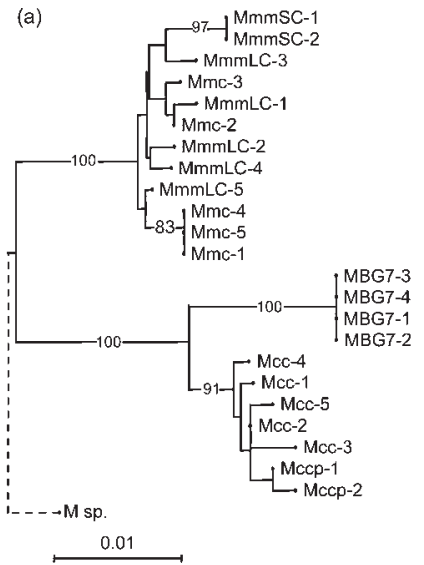

(b)

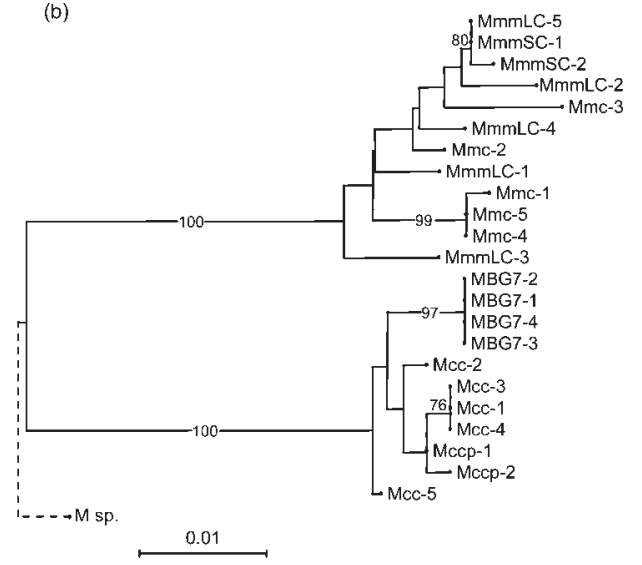

Fig. 3. Evolutionary distance trees based on fusA sequences of $561 \mathrm{bp} \mathrm{(a)} \mathrm{and} \mathrm{g/pO}$ sequences of $538 \mathrm{bp}$ (b). The trees were constructed using the neighbour-joining algorithm on a set of 23 strains belonging to the $M$. mycoides cluster. They are rooted on the grafting position of the uncharacterized strain 8756-C13 (labelled 'M sp.'), used as an outgroup. Bootstrap percentage values were calculated from 500 replications and values over $70 \%$ are displayed. Bars, distance equivalent to 1 substitution per 100 nucleotide positions. See Table 1 for strain details and Supplementary Table S1 for sequence accession numbers.
The two subclusters where clearly distinguished: M. mycoides subspecies on one side, M. capricolum subspecies and MBG7 on the other. Within the M. mycoides subcluster, the two MmmSC strains clustered together, though they were not neatly separated from the rest of the subcluster, where $\mathrm{MmmLC}$ and Mmc remained indistinguishable. As for the M. capricolum subgroup, Mccp strains could not be clearly separated from Mcc strains, whereas MBG7 constituted a distinct branch. All these main branches were supported by high bootstrap values (91-100\%).

Tree based on glpQ sequences. The general topology of the tree based on $g l p Q$ sequences (Fig. $3 \mathrm{~b}$ ) was similar to that based on fusA, with MmmSC strains more deeply embedded within the M. mycoides subcluster, being indistinguishable from the other strains.

Tree based on gyrB sequences. The tree inferred from gyrB sequences was incongruent with the phylogeny based on concatenated sequences (Fig. 4a). It differed from the fus $A$ and $g l p Q$ trees in the position of the MmmSC strains, which left the M. mycoides subcluster to join the $M$. capricolum subcluster ( $98 \%$ bootstrap). The MBG7 strains were attached to the $M$. capricolum subcluster, where Mccp constituted a separate branch. Independent analyses on each codon position revealed the same incongruent topology, and no influential units were identified among the 23 sequences. However, closer examination of variable nucleotide positions showed that the MmmSC strains shared several variant nucleotides with MBG7 strains, which attracted them towards the $M$. capricolum subcluster. These nucleotides were concentrated (7 of 10) in a short subsequence between positions 140 and 240 . Therefore, an analysis restricted to the second half of the sequence (Fig. $4 \mathrm{~b}$ ) agreed globally with the concatenated sequence phylogeny, with MmmSC placed in the $M$. mycoides subcluster.

Tree based on lepA sequences. The tree derived from lepA sequences (Fig. 5a) was largely incongruent with the common structure and did not allow a clear discrimination of the species M. mycoides and M. capricolum. As in the (a)

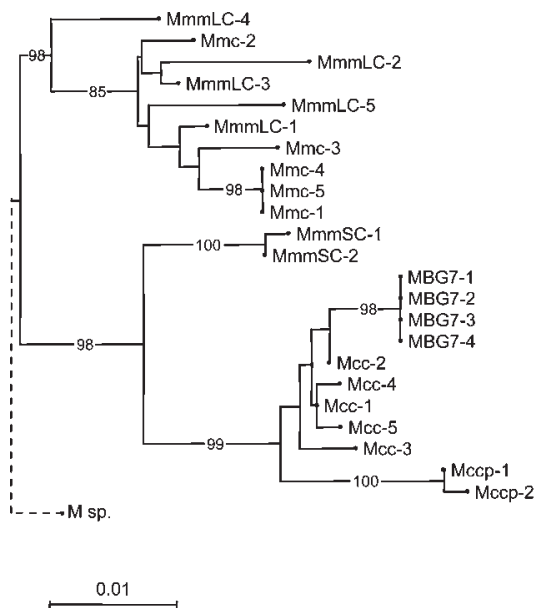

(b)

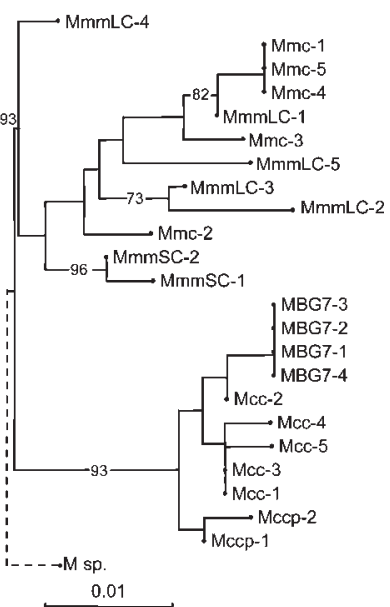

Fig. 4. Evolutionary distance trees based on gyrB sequences of $533 \mathrm{bp}$ (a) and based on the second half of these sequences from nucleotide position 267 (b). For further details refer to Fig. 3. See Table 1 for strain details and Supplementary Table S1 for sequence accession numbers. 
case of $g y r B$, the attraction between MmmSC and MBG7 was apparent, in this case inducing a separate group (74\% bootstrap) linked to the M. mycoides subcluster. The strains referred to as MmmLC-5 and MmmLC-3 were linked to the $M$. capricolum subcluster. These, as well as strain MmmLC-2, were detected as highly influential units, with Qd values over 0.22 , while the remaining values were below 0.12. MmmLC-2 and MmmLC-3 showed specific nucleotide variations, while MmmLC-5 shared variant nucleotides with the $M$. capricolum subcluster. In fact, the nine variable sites present in the first half of the lepA sequence of MmmLC-5 were characteristic of $M$. capricolum, whereas the second half contained seven variant nucleotides corresponding to a typical $M$. mycoides sequence. Analysis of the second half of this sequence (Fig. 5b) restored the three erratic strains to their subcluster but did not suppress the attraction between MmmSC and MBG7. When the analysis was restricted to the initial codon position (Fig. 5c), the common structure was recovered (except for strain MmmLC-5), with MmmSC linked to the M. mycoides subcluster and MBG7 to the $M$. capricolum subcluster, indicating that their shared nucleotide variations, as well as the specific variant nucleotides of the MmmLC-2 and MmmLC-3 sequences, mainly concerned the least-constrained codon positions.

Tree based on $r p \circ B$ sequences. The tree derived from $r p o B$ sequences (Fig. 6a) was highly incongruent with the common structure and neither of the two subclusters could be clearly discriminated. Analyses based on partial sequences and on each codon position did not allow this structure to be clarified. However, strain MmmLC-1, corresponding to strain $\mathrm{Y}_{\text {-goat }}{ }^{\mathrm{R}}$, was identified as a strongly influential unit ( $\mathrm{Qd}$ value of 0.2 , whereas the others did not exceed 0.02 ). In six variable positions, the sequence of strain $\mathrm{Y}_{\text {-goat }}{ }^{\mathrm{R}}$ shared variant nucleotides characteristic of $M$. capricolum, which were not shared by MBG7. This resulted in attraction of the $\mathrm{MmmLC} / \mathrm{Mmc}$ group towards the M. capricolum subspecies, accompanied by repulsion of MBG7. The tree constructed by exclusion of strain Y-goat ${ }^{\mathrm{R}}$ (Fig. 6b) restored an M. capricolum subcluster including MBG7 and an M. mycoides subcluster, though variant nucleotides specific to MmmSC, partly shared by strain MmmLC-4, kept them in a separate group.

\section{Phylogeny of the M. mycoides cluster and taxonomic implications}

The phylogeny of the M. mycoides cluster has been inferred from a set of five concatenated protein-coding gene sequences and strengthened by separate analyses performed on each individual gene. Although incongruences were observed for certain genes, which explain the ambiguities that have been found in previous studies, the 'mean structure' represented by the concatenated sequence tree could be recognized behind gene specificities when reinforced in this manner. This phylogeny does not contradict that based on 16S rRNA gene sequences (Pettersson et al., 1996). However, the reduced variability of the 16S rRNA gene sequence, added to the specific evolutionary processes of this genetic marker, has not allowed the close relationships existing between these organisms to be completely resolved: only 36 variable positions were found in a comparison of 1467 nucleotides corresponding to the sequences of the two rRNA operons of 10 strains of the $M$. mycoides cluster, including all type strains, increasing to 66 (30 unique positions) when $M$. putrefaciens $\mathrm{KS}^{\mathrm{T}}$ was included. The use of five housekeeping gene sequences has not only reduced the bias generated by using a single gene sequence but has also
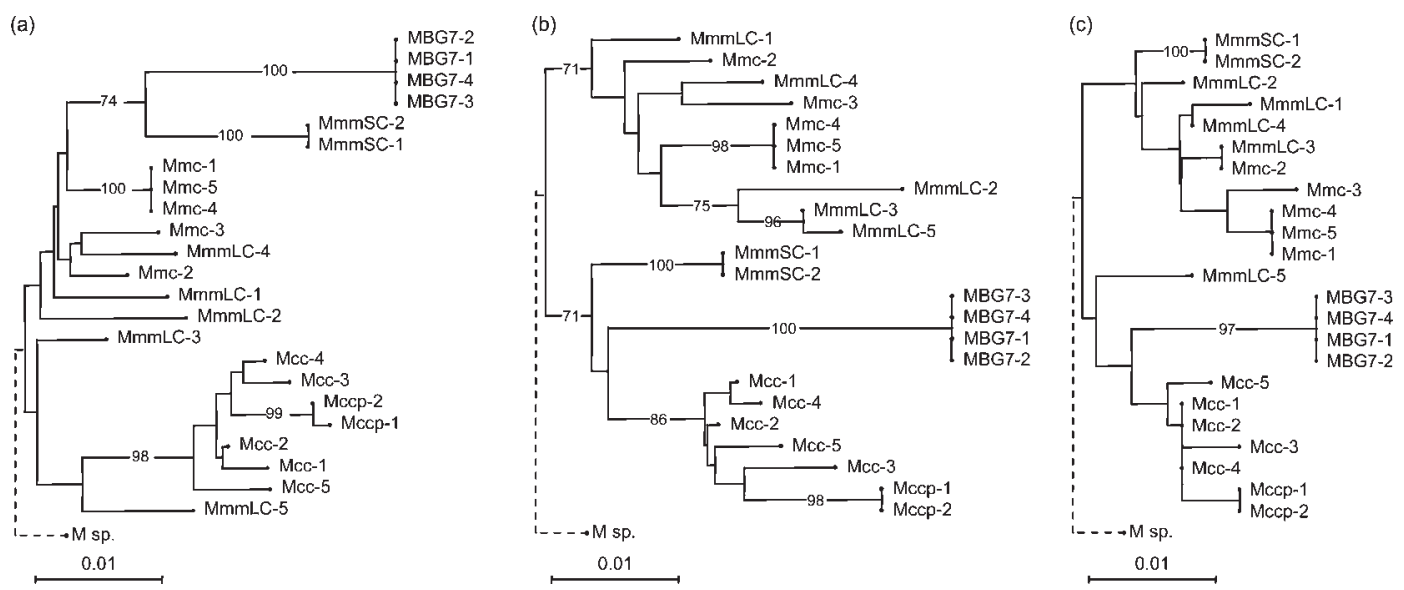

Fig. 5. Evolutionary distance trees based on lepA sequences of $657 \mathrm{bp}$ (a) and based on the second half of these lepA sequences, from nucleotide position $328(\mathrm{~b})$, and a tree inferred from the first codon positions of the 657 bp lepA sequences (c). For further details refer to Fig. 3. See Table 1 for strain details and Supplementary Table S1 for sequence accession numbers. 
(a)

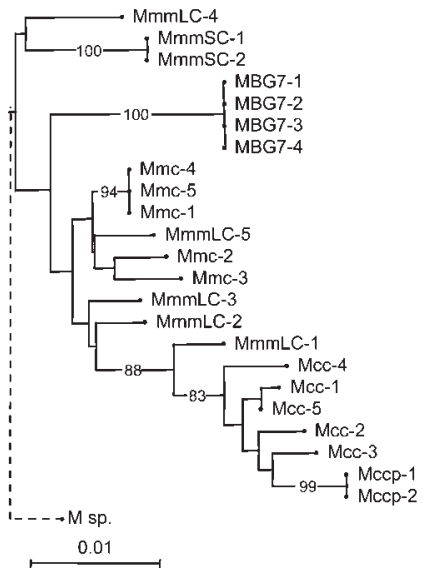

(b)

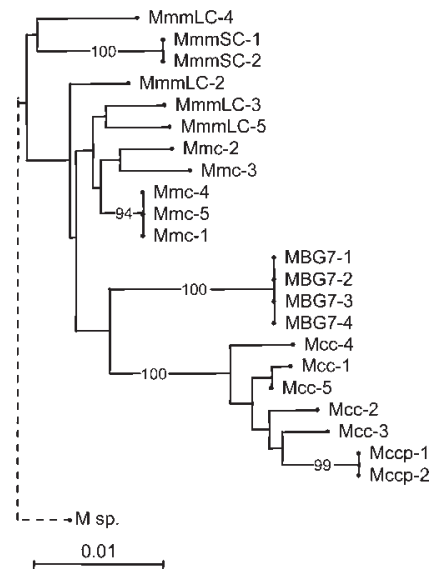

Fig. 6. Evolutionary distance trees based on $r p o B$ sequences of $625 \mathrm{bp}$ constructed from the set of 23 strains belonging to the $M$. mycoides cluster (a) and constructed as above but excluding strain MmmLC-1 (b). For further details refer to Fig. 3. See Table 1 for strain details and Supplementary Table S1 for sequence accession numbers. provided greater resolution by considerably increasing variability. The partial gene sequences used for phylogeny inference provided 106-185 variable positions in a comparison of the 28 strains analysed and 36-68 when limiting the comparison to the 23 strains of the $M$. mycoides cluster (Table 3). Concatenation of the five sequences increased the number of variable positions to 261, of which 203 were informative for phylogenetic analysis of the M. mycoides cluster.

This approach has allowed us to construct a reliable tree to assign phylogenetic positions to all members of the $M$. mycoides cluster, showing once more that the existing taxonomy needs to be reviewed.

Phylogenetic position of Mycoplasma sp. bovine group 7 of Leach. The greatest incongruences, found in two of the five gene sequences analysed in this study, resulted from the attraction between MmmSC and MBG7. For gyrB, this proximity was limited to a subsequence and, in the case of lepA, it essentially concerned the least-constrained codon positions. The common structure corresponding to fus $A$ and $g l p Q$ (and $r p o B$ when excluding the influential unit MmmLC-1), which places MmmSC in the $M$. mycoides subcluster and MBG7 in the M. capricolum subcluster, is not strongly contradicted by analyses of the other two genes and can therefore be retained. Furthermore, the partial attraction observed between MBG7 and MmmSC explains the ambiguity that persists in the present classification and why, unlike the other members of the M. mycoides cluster, the group of strains referred to as MBG7 has not yet been assigned a species name. Investigations based on DNA-DNA hybridization (Christiansen \& Ernø, 1982), identification with a DNA probe (Taylor et al., 1992) and electrophoretic analysis of isoenzymes and serology (Salih et al., 1983) have suggested the classification of MBG7 as a distinct species. DNA-DNA hybridization studies by Askaa et al. (1978), lipoprotein sequence similarity and immunological cross-reactions (Frey et al., 1998), as well as sequence similarity of the glycerol transport locus gtsABC (Djordjevic et al., 2003), have revealed a close relatedness to $\mathrm{MmmSC}$. In contrast, serological cross-reactions instead suggested that MBG7 was closer to the M. capricolum subspecies (Cottew et al., 1987; Ernø et al., 1983), which was confirmed by 1D and 2D SDS-PAGE profile comparisons (Costas et al., 1987; Olsson et al., 1990). In addition, MSC4, a small stable RNA, was found to be specific to the species $M$. capricolum and MBG7 (Ushida et al., 2003), and recent phylogenetic studies based on 16S rRNA gene sequences (Pettersson et al., 1996), 16S-23S rRNA intergenic spacer sequences (Harasawa et al., 2000) and a putative membrane protein gene (Thiaucourt et al., 2000) all grouped MBG7 in the $M$. capricolum subcluster. The results presented here provide additional evidence regarding the genetic identification of this group of strains and support its classification as a subspecies of $M$. capricolum.

Interestingly, all strains of MBG7 provided identical sequences for each of the five genes analysed. This seems to indicate a very limited intraspecies variability for this group of strains and, presumably, a recent differentiation within the species $M$. capricolum.

All members of the M. mycoides cluster have been isolated from small ruminants (Atalaia et al., 1987; Kusiluka et al., 2001), where multiple infections are frequent (Cottew \& Yeats, 1982). These conditions may well promote horizontal gene transfer between the different species. Genomic exchanges were suspected for several strains analysed in this study. This is the case for strain MmmLC-5, for which the first half of the lepA sequence was characteristic of $M$. capricolum while the second half corresponded to a typical M. mycoides sequence. A lateral transfer of genes or gene fragments may explain the local proximity that exists between MBG7 and MmmSC that has been observed previously and is again shown in two of the five genes analysed in this work.

Relatedness of $M$. mycoides subsp. mycoides LC and $M$. mycoides subsp. capri. The distinction of MmmLC and 
Mmc as separate subspecies has been questioned for some time. Numerous investigations have provided evidence to suggest that they may be grouped as a single subspecies within the M. mycoides subcluster. Although early DNADNA hybridization studies by Askaa et al. (1978) suggested that Mmc was closer to MmmSC than to MmmLC, analyses of 1D and 2D PAGE protein patterns proved the opposite (Costas et al., 1987; Leach et al., 1989; Olsson et al., 1990; Rodwell, 1982; Salih et al., 1983). Identification using a DNA probe (Taylor et al., 1992) and analysis of LppA sequence similarity and serological cross-reactions (Monnerat et al., 1999) also suggested a very close relatedness between MmmLC and Mmc, and two phylogenetic studies of the M. mycoides cluster concluded that they should be grouped into a single entity (Pettersson et al., 1996; Thiaucourt et al., 2000). However, the International Committee on Systematics of Prokaryotes Subcommittee on the taxonomy of Mollicutes has indicated that additional comparative analysis is required in order to clarify this matter (Johansson \& Bradbury, 2007).

Additional evidence has recently been presented by Vilei et al. (2006) based on rpoB sequence comparisons. In the present work, comparisons have been extended to five genes using a similar sample of strains, though strain WK354, included as an Mmc strain in previous studies (Monnerat et al., 1999; Thiaucourt et al., 2000), was characterized in this work as MmmLC by both growth inhibition and dot immunobinding techniques. As in previous studies, the data obtained in this work show that MmmLC is closer to Mmc than to the biotype MmmSC and that MmmLC and Mmc are not sufficiently distant to constitute separate entities. Furthermore, neither of these two organisms represents the aetiological agent of a characteristic disease, which may have been a justification for their separation. For example, this had been the rationale for the establishment of Mccp as a separate subspecies of $M$. capricolum.

In conclusion, MmmLC and Mmc appear to constitute a single entity, which could justify their combination into the same taxonomic subspecies.

Position of $M$. putrefaciens and other related species. $M$. putrefaciens is not included in the classical M. mycoides cluster and may be easily differentiated from the members of this cluster by biochemical and serological methods (Tully et al., 1974) as well as by a specific PCR test (Peyraud et al., 2003). However, on the basis of $16 \mathrm{~S}$ rRNA gene sequence similarity, it was included in the phylogenetic M. mycoides cluster, within the Spiroplasma group (Weisburg et al., 1989). Also based on $16 \mathrm{~S}$ rRNA sequence data, the closely related species $M$. cottewii and $M$. yeatsii were included in this phylogenetic cluster, branching with $M$. putrefaciens (Heldtander et al., 1998). Although the latter authors suggested that these three species should actually constitute a separate cluster, their precise taxonomic position remains uncertain. In this study, M. putrefaciens, M. cottewii and M. yeatsii were distant from the members of the classical cluster, with an important number of strictly specific nucleotide variations, confirming that they should not be included in the M. mycoides cluster.

\section{Application to identification and classification of strains}

The great relevance of diseases such as CBPP and CCPP and the fact that several members of the M. mycoides cluster produce similar clinical signs in small ruminants with different virulence mean that precise identification is of paramount importance. However, some of these mycoplasmas are difficult to differentiate by conventional techniques (Cottew et al., 1987). Growth inhibition, which is often used for species identification, is frequently hampered both by interspecies cross-reactions and by intraspecies variability. Cross-reactions are frequent between biotypes MmmSC and MmmLC (Cottew \& Yeats, 1978; Thigpen et al., 1983) and between MBG7 and Mccp (Cottew et al., 1987; Ernø et al., 1983; Kibe et al., 1985), hindering confirmation of CBPP and CCPP, respectively. A high degree of intraspecies heterogeneity has also been shown using several techniques, particularly within $\mathrm{MmmLC} / \mathrm{Mmc}$ and also Mcc to a certain extent, whereas MmmSC and Mccp appear rather homogeneous (Abu-Groun et al., 1994; Christiansen \& Ernø, 1990; Costas et al., 1987; Kusiluka et al., 2001). Our data correlate with these observations and emphasize once more the limitations encountered in the classification of certain strains and, furthermore, in the definition and delimitation of species and subspecies within the M. mycoides cluster.

Given the increased resolving power of the strategy presented in this work, it was hypothesized that it might allow strains that have proved difficult to affiliate to be classified. This is the case for strain 8756-C13, isolated from a Rocky Mountain goat, for which identification by classical methods is challenging. Cultural parameters indicated that it might be an MmmLC strain, though growth-inhibition tests were negative for both MmmLC and Mmc. It was finally classed as MmmLC on the basis of immunofluorescence results, although electrophoretic patterns were inconclusive. The data from this study show that this is not a typical strain of the M. mycoides cluster, but a nearby relative, located closer to this cluster than to other related species such as $M$. putrefaciens, M. cottewii and $M$. yeatsii. Presumably, study of new isolates originating from diverse geographical and host origins will result in the identification of further 'atypical' strains related to this cluster and, until whole-genome sequence comparisons become accessible to diagnostic laboratories, protein gene sequence data may prove to be a good addition to $16 \mathrm{~S}$ rRNA genes for fine strain characterization. However, analyses based on multiple gene sequences are still difficult to use for routine diagnostic purposes. On the other hand, fusA may be an interesting alternative for rapid identification and phylogenetic positioning by PCR and sequencing, for which the set of sequences presented in this work may 
serve as a reference: although variability was low among the fusA sequences from M. mycoides strains, the tree inferred from this gene was very similar to that based on the concatenated sequences.

\section{Concluding remarks}

Conserved protein-coding gene sequences may be used to complement and extend 16S rRNA gene sequence data to allow differentiation of closely related groups of organisms, such as the members of the M. mycoides cluster. However, a single gene tree can be largely incongruent with the species tree, which represents its true evolutionary history. Therefore, several genes must be analysed simultaneously. It is proposed here to consider the tree inferred from five concatenated gene sequences as a reliable estimation of the species phylogeny, since this 'mean structure' can be detected in each individual gene tree, even if it is partially masked by specific gene evolution.

This approach has resulted in the construction of a reliable phylogenetic tree, which will be a universal tool for the classification of strains of the M. mycoides cluster, including those difficult to identify by classical methods. Furthermore, it may lead to the development of new techniques for rapid species identification and molecular epidemiology.

\section{ACKNOWLEDGEMENTS}

We are grateful to Valerie Barbe from Genoscope (Evry, France) for the results of MmmLC strain 95010-C1 whole-genome sequencing and to François Poumarat and Florence Tardy from AFSSA (Lyon, France) for characterization of the strains by dot immunobinding. We also thank Alan Blanchard and Pascal Sirand-Pugnet (INRABordeaux, France) for invaluable discussions and are indebted to Janet Bradbury for critical reading of the manuscript and for her precious suggestions.

\section{REFERENCES}

Abu-Groun, E. A. M., Taylor, R. R., Varsani, H., Wadher, B. J., Leach, R. H. \& Miles, R. J. (1994). Biochemical diversity within the 'Mycoplasma mycoides cluster'. Microbiology 140, 2033-2042.

Askaa, G., Ernø, H. \& Ojo, M. O. (1978). Bovine mycoplasmas: classification of groups related to Mycoplasma mycoides. Acta Vet Scand 19, 166-178.

Atalaia, V., Machado, M. \& Franco Frazão, F. (1987). Patologia dos pequenos ruminantes infecções em ovinos e caprinos, originadas pelo micoplasma do grupo 7, Leach (Pg. 50). Repos Trab Lab Nac Invest Vet 19, 55-60 (in Portuguese).

Christiansen, C. \& Ernø, H. (1982). Classification of the F38 group of caprine mycoplasma strains by DNA hybridization. J Gen Microbiol 128, 2523-2526.

Christiansen, G. \& Ernø, H. (1990). RFLP in rRNA genes of Mycoplasma capricolum, the caprine F38-like group and the bovine serogroup 7. Zentralbl Bakteriol Hyg Suppl 20, 479-488.

Clyde, W. A., Jr (1983). Growth inhibition tests. In Methods in Mycoplasmology, pp. 405-410. Edited by S. Razin \& J. G. Tully. New York: Academic Press.
Cordy, D. R., Adler, H. E. \& Yamamoto, R. (1955). A pathogenic pleuropneumonialike organism from goats. Cornell Vet 45, 50-68.

Costas, M., Leach, R. H. \& Mitchelmore, D. L. (1987). Numerical analysis of PAGE protein patterns and the taxonomic relationships within the 'Mycoplasma mycoides cluster'. J Gen Microbiol 133, 3319-3329.

Cottew, G. S. \& Yeats, F. R. (1978). Subdivision of Mycoplasma mycoides subsp. mycoides from cattle and goats into two types. Aust Vet J 54, 293-296.

Cottew, G. S. \& Yeats, F. R. (1981). Occurrence of mycoplasmas in clinically normal goats. Aust Vet J 57, 52-53.

Cottew, G. S. \& Yeats, F. R. (1982). Mycoplasmas and mites in the ears of clinically normal goats. Aust Vet J 59, 77-81.

Cottew, G. S., Breard, A., DaMassa, A. J., Ernø, H., Leach, R. H., Lefevre, P. C., Rodwell, A. W. \& Smith, G. R. (1987). Taxonomy of the Mycoplasma mycoides cluster. Isr J Med Sci 23, 632-635.

DaMassa, A. J., Tully, J. G., Rose, D. L., Pitcher, D., Leach, R. H. \& Cottew, G. S. (1994). Mycoplasma auris sp. nov., Mycoplasma cottewii sp. nov., and Mycoplasma yeatsii sp. nov., new sterol-requiring mollicutes from the external ear canals of goats. Int J Syst Bacteriol 44, 479-484.

Djordjevic, S. P., Vilei, E. M. \& Frey, J. (2003). Characterization of a chromosomal region of Mycoplasma sp. bovine group 7 strain PG50 encoding a glycerol transport locus (gtsABC). Microbiology 149, 195-204.

Ernø, H., Leach, R. H., Salih, M. M. \& MacOwan, K. J. (1983). The F38like group, a new group of caprine mycoplasmas? Acta Vet Scand 24, 275-286.

Frey, J., Cheng, X., Monnerat, M. P., Abdo, E. M., Krawinkler, M., Bölske, G. \& Nicolet, J. (1998). Genetic and serological analysis of the immunogenic 67-kDa lipoprotein of Mycoplasma sp. bovine group 7. Res Microbiol 149, 55-64.

Harasawa, R., Hotzel, H. \& Sachse, K. (2000). Comparison of the 16S-23S rRNA intergenic spacer regions among strains of the Mycoplasma mycoides cluster, and reassessment of the taxonomic position of Mycoplasma sp. bovine group 7. Int J Syst Evol Microbiol 50, 1325-1329.

Heldtander, M., Pettersson, B., Tully, J. G. \& Johansson, K.-E. (1998). Sequences of the $16 \mathrm{~S}$ rRNA genes and phylogeny of the goat mycoplasmas Mycoplasma adleri, Mycoplasma auris, Mycoplasma cottewii and Mycoplasma yeatsii. Int J Syst Bacteriol 48, 263-268.

Johansson, K.-E. \& Bradbury, J. M. (2007). International Committee on Systematics of Prokaryotes Subcommittee on the taxonomy of Mollicutes. Minutes of the meetings, 7 and 12 July 2002, Vienna, Austria. Int J Syst Evol Microbiol 57, 181-186.

Kibe, M. K., Bidwell, D. E., Turp, P. \& Smith, G. R. (1985). Demonstration of cross-reactive antigens in F38 and related mycoplasmas by enzyme-linked immunosorbent assay (ELISA) and immunoblotting. J Hyg (Lond) 95, 95-106.

Kim, K. S., Ko, K. S., Chang, M. W., Hahn, T. W., Hong, S. K. \& Kook, Y. H. (2003). Use of $r p o B$ sequences for phylogenetic study of Mycoplasma species. FEMS Microbiol Lett 226, 299-305.

Kumar, S., Tamura, K. \& Nei, M. (2004). MEGA3: integrated software for molecular evolutionary genetics analysis and sequence alignment. Brief Bioinform 5, 150-163.

Kusiluka, L. J., Ojeniyi, B., Friis, N. F., Kokotovic, B. \& Ahrens, P. (2001). Molecular analysis of field strains of Mycoplasma capricolum subspecies capripneumoniae and Mycoplasma mycoides subspecies mycoides, small colony type isolated from goats in Tanzania. Vet Microbiol 82, 27-37. 
Laws, L. (1956). A pleuropneumonia-like organism causing peritonitis in goats. Aust Vet J 32, 326-329.

Leach, R. H. (1967). Comparative studies of mycoplasma of bovine origin. Ann N Y Acad Sci 143, 305-316.

Leach, R. H., Costas, M. \& Mitchelmore, D. L. (1989). Relationship between Mycoplasma mycoides subsp. mycoides ('large-colony' strains) and M. mycoides subsp. capri, as indicated by numerical analysis of one-dimensional SDS-PAGE protein patterns. J Gen Microbiol 135, 2993-3000.

Leach, R. H., Ernø, H. \& MacOwan, K. J. (1993). Proposal for designation of F38-type caprine mycoplasmas as Mycoplasma capricolum subsp. capripneumoniae subsp. nov. and consequent obligatory relegation of strains currently classified as $M$. capricolum (Tully, Barile, Edward, Theodore, and Ernø 1974) to an additional new subspecies, M. capricolum subsp. capricolum subsp. nov. Int J Syst Bacteriol 43, 603-605.

Miserez, R., Pilloud, T., Cheng, X., Nicolet, J., Griot, C. \& Frey, J. (1997). Development of a sensitive nested PCR method for the specific detection of Mycoplasma mycoides subsp. mycoides SC. Mol Cell Probes 11, 103-111.

Monnerat, M. P., Thiaucourt, F., Poveda, J. B., Nicolet, J. \& Frey, J. (1999). Genetic and serological analysis of lipoprotein LppA in Mycoplasma mycoides subsp. mycoides LC and Mycoplasma mycoides subsp. capri. Clin Diagn Lab Immunol 6, 224-230.

Olsson, B., Bölske, G., Bergström, K. \& Johansson, K. E. (1990). Analysis of caprine mycoplasmas and mycoplasma infections in goats using two-dimensional electrophoresis and immunoblotting. Electrophoresis 11, 861-869.

Perrier, X., Flori, A. \& Bonnot, F. (2003). Data analysis methods. In Genetic Diversity of Cultivated Tropical Plants, pp. 43-76. Edited by P. Hamon, M. Seguin, X. Perrier \& J. C. Glaszmann. Enfield, UK: Science Publishers.

Pettersson, B., Leitner, T., Ronaghi, M., Bölske, G., Uhlén, M. \& Johansson, K.-E. (1996). Phylogeny of the Mycoplasma mycoides cluster as determined by sequence analysis of the 16S rRNA genes from the two rRNA operons. J Bacteriol 178, 4131-4142.

Peyraud, A., Woubit, S., Poveda, J. B., De la Fe, C., Mercier, P. \& Thiaucourt, F. (2003). A specific PCR for the detection of Mycoplasma putrefaciens, one of the agents of the contagious agalactia syndrome of goats. Mol Cell Probes 17, 289-294.

Poumarat, F., Longchambon, D. \& Martel, J. L. (1992). Application of dot immunobinding on membrane filtration (MF dot) to the study of relationships within "M. mycoides cluster" and within "glucose and arginine-negative cluster" of ruminant mycoplasmas. Vet Microbiol 32, 375-390.

Rodwell, A. W. (1982). Emmy Klieneberger-Nobel Award Lecture: The protein fingerprints of mycoplasmas. Rev Infect Dis 4 (Suppl.), S8-S17.
Rokas, A., Williams, B. L., King, N. \& Carroll, S. B. (2003). Genomescale approaches to resolving incongruence in molecular phylogenies. Nature 425, 798-804.

Saitou, N. \& Nei, M. (1987). The neighbor-joining method: a new method for reconstructing phylogenetic trees. Mol Biol Evol 4, 406-425.

Salih, M. M., Ernø, H. \& Simonsen, V. (1983). Electrophoretic analysis of isoenzymes of mycoplasma species. Acta Vet Scand 24, 14-33.

Santos, S. R. \& Ochman, H. (2004). Identification and phylogenetic sorting of bacterial lineages with universally conserved genes and proteins. Environ Microbiol 6, 754-759.

Schwan, T. G., Raffel, S. J., Schrumpf, M. E., Policastro, P. F., Rawlings, J. A., Lane, R. S., Breitschwerdt, E. B. \& Porcella, S. F. (2005). Phylogenetic analysis of the spirochetes Borrelia parkeri and Borrelia turicatae and the potential for tick-borne relapsing fever in Florida. J Clin Microbiol 43, 3851-3859.

Simmons, G. C. \& Johnston, L. A. Y. (1963). Arthritis in calves caused by Mycoplasma species. Aust Vet J 39, 11-14.

Taylor, T. K., Bashiruddin, J. B. \& Gould, A. R. (1992). Relationships between members of the Mycoplasma mycoides cluster as shown by DNA probes and sequence analysis. Int J Syst Bacteriol 42, 593-601.

Thiaucourt, F. \& Bölske, G. (1996). Contagious caprine pleuropneumonia and other pulmonary mycoplasmoses of sheep and goats. Rev Sci Tech 15, 1397-1414.

Thiaucourt, F., Lorenzon, S., David, A. \& Breard, A. (2000). Phylogeny of the Mycoplasma mycoides cluster as shown by sequencing of a putative membrane protein gene. Vet Microbiol 72, 251-268.

Thigpen, J. E., Cottew, G. S., Yeats, F., McGhee, C. E. \& Rose, D. L. (1983). Growth characteristics of large- and small-colony types of Mycoplasma mycoides subsp. mycoides on $5 \%$ sheep blood agar. J Clin Microbiol 18, 956-960.

Tully, J. G., Barile, M. F., Edward, D. G. ff., Theodore, T. S. \& Ernø, H. (1974). Characterization of some caprine mycoplasmas, with proposals for new species, Mycoplasma capricolum and Mycoplasma putrefaciens. J Gen Microbiol 85, 102-120.

Ushida, C., Yoshida, A., Miyakawa, Y., Ara, Y. \& Muto, A. (2003). Distribution of the MCS4 RNA genes in mycoplasmas belonging to the Mycoplasma mycoides cluster. Gene 314, 149-155.

Vilei, E. M., Korczak, B. M. \& Frey, J. (2006). Mycoplasma mycoides subsp. capri and Mycoplasma mycoides subsp. mycoides LC can be grouped into a single subspecies. Vet Res 37, 779-790.

Weisburg, W. G., Tully, J. G., Rose, D. L., Petzel, J. P., Oyaizu, H., Yang, D., Mandelco, L., Sechrest, J., Lawrence, T. G. \& other authors (1989). A phylogenetic analysis of the mycoplasmas: basis for their classification. J Bacteriol 171, 6455-6467.

Woese, C. R. (1987). Bacterial evolution. Microbiol Rev 51, 221-271. 